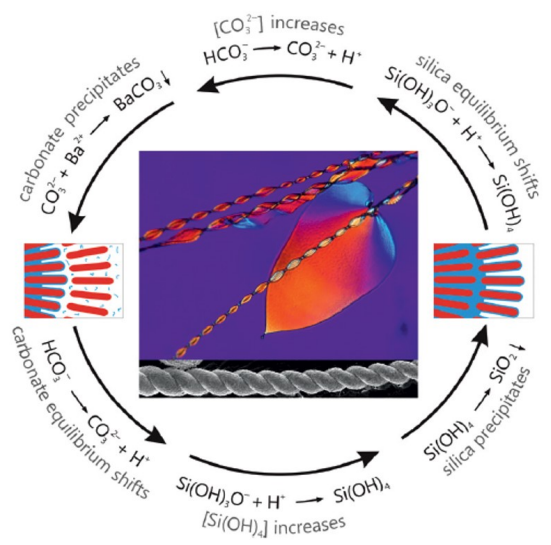

Figure 1.

Keywords: Biomorphs, biomimetic materials, crystallization, silica, carbonate

\section{MS14-O3 Biomimetic formation of calcite in intermixed gelatin/agarose hydrogels: Aggregate co-orientation and $\mathrm{Mg}$ content}

Xiaofei Yin $^{1}$, Fitriana Nindiyasari ${ }^{1}$, Erika Griesshaber ${ }^{1}$, Lurdes Fernández-Díaz ${ }^{2,3}$, Andreas Ziegler ${ }^{4}$, Wolfgang W. Schmahl ${ }^{1}$

1. Department für Geo- und Umweltwissenschaften, Ludwig-Maximilians-Universität, 80333 Munich, Germany

2. Departamento de Cristalografía y Mineralogía, Universidad Complutense de Madrid, 28040 Madrid, Spain

3. Instituto de Geociencias (UCM, CSIC). C/ José Antonio Novais 2, 28040 Madrid, Spain

4. Central Facility for Electron Microscopy, University of Ulm, 89069 Ulm, Germany

email: xiaofei.yin@campus.lmu.de

Crystal aggregation and orientation in carbonate biological hard tissues such as shells and teeth is organic matrix mediated, a structure consisting of membranes (1A, star) and fibrils (1A, arrow). The gel fibril network creates pores where local crystallization environments resemble those formed by extracellular, biological matrices. In previous studies we investigated in single gel systems the effect of agarose and gelatin on calcite nucleation and aggregate formation ${ }^{[1]}$. Gel was always occluded into the aggregate and imposed a marked effect on crystal co-orientation and aggregate formation. In $\mathrm{Mg}$-free growth environments, either single crystals or radial aggregates with very few subunits formed. Was $\mathrm{Mg}$ added to the growth medium, the amount of internal boundaries increased as well as the number of subunits in the aggregate.

In this paper we discuss the effect on calcite nucleation, growth and aggregate formation of MIXED hydrogel systems. A very first attempt to use a mixture of hydrogels was conducted by Simon et $a l^{[2]}$. In our experiments we use agarose/gelatine mixtures to crystallize $\mathrm{CaCO}_{3}$ using the double-diffusion method. Mineral microstructure and texture is determined with high-resolution EBSD, the occlusion of gels and characteristics of their distribution is visualized with an FE-SEM on microtome polished, etched and critical point dried sample surfaces. In gel/mineral composites grown in 1:1 agarose/gelatin hydrogels (1B), the hydrogel fabric is characterized by thick fibrils, a high variety of pore sizes and numerous walls. Hydrogels in gel/mineral composites grown in 2:1 agarose/gelatin gels (1C) are dense and consist of thick fibrils but also gel membranes (1C, stars and rectangles). When hydrogels with a $1: 1$ agarose:gelatin ratio are used (1B), calcite grows as hopper crystals bounded by terraced $\{104\}$ faces. In an agarose:gelatin ratio of $2: 1$ used (1C), calcite crystals appear elongated along their $c$-axis and bounded by extremely terraced very rough and curved (104) surfaces that seem to correspond to steep rhombohedra. In both cases, crystals consist of rhombohedral sub-blocks (size < $1 \mu \mathrm{m})$ arranged in an approximately equal orientation. Differences in the occluded gel fabric are the consequence of hydrogel strength and crystallization pressure.

[1] F. Nindiyasari et al., Cryst. Growth Des.15, 2667-2685, 2015

[2] P. Simon et al., Eur. J. Inorg. Chem. 35, 5370-5377, 201 


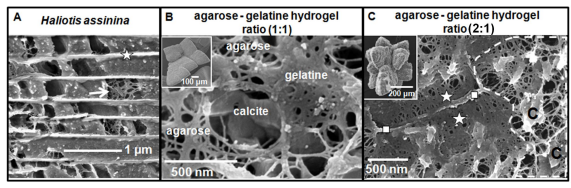

Figure 1. Characteristics of extracellular carbonate hard tissue (1A) and hydrogel (1B, 1C) matrices.

Keywords: EBSD, calcite-hydrogel aggregate, extracellular matrix, gelatin, agarose

\section{MS14-04 Polar ordering of macromolecular chains in biomimetic composite materials and natural tissues

\author{
Martin Sommer ${ }^{1}$, Matthias Burgener ${ }^{1}$, Hanane Aboulfadl ${ }^{1}$, Jürg
} Hulliger $^{1}$}

1. Department of Chemistry and Biochemistry, University of Bern, Freiestrasse 3, CH-3012 Bern, Switzerland

email: martin.sommer@dcb.unibe.ch

The state of alignment of macromolecules in biomimetic materials and natural tissues will be discussed by investigating a mechanism of electrical polarity formation: An in vitro grown biomimetic fluorapatite (FAp)/ gelatin composite is analyzed for its polar properties by second harmonic (SHGM) and scanning pyroelectric microscopy (SPEM). Growth media containing biological macromolecules do not only influence the morphology, but can also have a significant impact on the polarity of a composite [1]. Hexagonal prismatic seed crystals of FAp formed in gelatin represent a monodomain polar state due to aligned mineralized gelatin molecules [1]. Later growth stages, expressing a dumbbell morphology, develop into a bipolar state because of surface recognition by gelatin functionality.

Other inorganic materials like $\mathrm{CaCO}_{3}$ (calcite), $\mathrm{CaSO}_{4}$ and $\mathrm{CaC}_{2} \mathrm{O}_{4}$ formed analogous composites when grown in a gelatin matrix. In addition, all of these composites revealed a similar behavior regarding polarity formation as compared to FAp. Subsequently the gelatin matrix was replaced by other gels, such as agar-agar and carrageenan, which developed the same kind of bipolar state. By growing the inorganic components in tetramethylorthosilicate (TMOS, nonpolar gel), SPEM experiments did not reveal any polarity. In all grown composites, the only present organic and polar material are the polar gel macromolecules. Single crystals of the investigated inorganic components, e.g. FAp and $\mathrm{CaCO}_{3}$, are centrosymmetric. Therefore, the only possible origin of polarity in these biomimetic composites is due to the incorporated macromolecules.

Comparing SPEM data of natural hard tissues (teeth and bone) with biomimetic FAp/ gelatin, calcite/ gelatin and other investigated composites, a surprising analogy in view of growth-induced states of polarity is found: The development of polarity in vivo and in vitro can be explained by a Markov-type mechanism of molecular recognition during the attachment of macromolecules. Furthermore, SHGM was able to reveal the polar structure of tissues by the application of phase sensitive experiments and the use of a polar reference crystal [2].

[1] Burgener, M.; Putzeys, T.; Gasthi, M. P.; Busch, S.; Aboulfadl, H.; Wübbenhorst, M.; Kniep, R.; Hulliger, J. Biomacromolecules 2015, 16, 2814-2819.

[2] Aboulfadl, H.; Hulliger, J. J. Struct. Biol. 2015, $192,67-75$.

Keywords: polarity, polarity formation, biomimetic, composite materials, natural tissues 